\title{
Research Paper: Effectiveness of Action Potential Simulation and Transcutaneous Electrical Nerve Stimulation on Pain and Function of Patients With Chronic Mechanical Shoulder Impairment
}

\author{
Ahmad Reza Askary Ashtiani' ${ }^{1}$ Fateme Ghiasi ${ }^{2 *}$, Kheir Mohammad Noraie ${ }^{3}$, Bahareh Khodadadi Bohloli ${ }^{2}$ \\ 1. Health Promotion Research Center, Zahedan University of Medical Sciences, Zahedan, Iran. \\ 2. Department of Physiotherapy, School of Rehabilitation Sciences, Zahedan University of Medical Sciences, Zahedan, Iran. \\ 3. Department of Physical Education, Faculty of Human Science, Saravan Branch, Islamic Azad University, Saravan, Iran.
}

Citation: Askary Ashtiani AR, Ghiasi F, Noraie KM, Khodadadi Bohloli B. Effectiveness of Action Potential Simulation and Transcutaneous Electrical Nerve Stimulation on Pain and Function of Patients With Chronic Mechanical Shoulder Impairment. Physical Treatments. 2016; 6(2):79-84. https://doi. org/10.18869/NRIP.PTJ.6.2.79

doi : https://doi.org/10.18869/NRIP.PTJ.6.2.79

Article info:

Received: 05 Feb. 2016

Accepted: 02 Jun. 2016
Keywords:

Action potential Stimulation, TENS, Pain, Function, Mechanical shoulder impairment

\begin{abstract}
A B S T R A C T
Purpose: Rehabilitation and physiotherapy have a major role in reducing the pain and increasing the functional ability in patients with shoulder joint pain. This study aimed to evaluate the effect of Action Potential Simulation (APS) and Transcutaneous Electrical Nerve Stimulation (TENS) in patients with Chronic Mechanical Shoulder Pain (CMSP).
\end{abstract}

Methods: In this quasi-experimental study, 32 patients with CMSP were enrolled. Patients were randomly assigned into APS group and TENS group, both of them received 6 sessions of intervention. The study instruments included pain intensity and shoulder Range Of Motion (ROM), America Surgeons Standardized Shoulder Assessment Form, and Western Ontario Rotator Cuff Index. The stability of scapula and shoulder function (shoulder dyskinesia) were measured before and after the intervention. The obtained data were analyzed using $t$ tests by SPSS19 $(\mathrm{P}<0.05)$.

Results: Pain intensity, shoulder ROM, and stability of scapula decreased in both groups. However, there were no significant differences between groups $(\mathrm{P}>0.05)$. In addition, shoulder function did not change after intervention in both groups $(\mathrm{P}>0.05)$.

Conclusion: With regard to study findings, APS and TENS are effective in pain reduction but none has preference over the other in treatment approaches.

\section{Introduction}

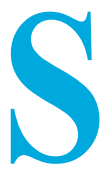

houlder is one of the most complex and important joints in the body. Normal function of shoulder joint is essential for hands movement in space and easy access to objects and taking different spatial positions [1]. Because of shoulder joint overuse in daily activities, its complicated biomechanics, and sensitive surrounding structures, the impairment of this joint is prevalent. Consequently, most patients re-

* Corresponding Author:

Fateme Ghiasi, PhD

Address: Department of Physiotherapy, School of Rehabilitation Sciences, Zahedan University of Medical Sciences, Dr. Hesabi Sq., Zahedan, Iran. Phone: +98 (54) 33424675

E-mail:fghiasi_p@yahoo.com 
ferring to physical therapy clinics are complaining from shoulder pain [2-4].

Besides pharmacological treatments for musculoskeletal injuries, rehabilitation and physical therapy have a leading role in pain reduction and functional improvement of these patients [5]. Common electrical modalities, like Transcutaneous Electrical Nerve Stimulation (TENS), with the main mechanism of activation of A-beta neural fibers - a mechanoreceptor with low threshold of skin stimulation - depresses neural pain afferent fibers irritability in spinal cord (C, A-delta). This phenomenon might act through pain gait control mechanism [6]. From perspective of the modern science, electrotherapy has specific place in physical therapy approaches. Furthermore, investigating the knowledge of practical and theoretical principles of this practice enables physical therapists to alleviate patients' pain and restore their abilities.

One of the most novel methods for pain reduction in physical therapy is Action Potential Simulation (APS) therapy. APS was invented in 1992 and has extensive use for reduction of chronic pain [7, 8]. APS current is the combination of rectifier and alternative currents, which simulates natural provocation of nerve conduction and is unique in this respect [9]. Electrical potential of each cell in normal state is completely specific and stable but variable in abnormal state quantity. In medicine, definition of amount of normal electrical potential and type of disease and its severity are determined with respect to deviation from this scale. Therefore, the treatment aims to convert the abnormal potential to normal one [9]. APS has different pulse wave compared to TENS and its designers claim several physiological effects for it like increasing leucine, encephalin, plasma melatonin, and tissue adenosine triphosphate (ATP); reducing plasma beta endorphin; and dilating local blood vessels which all lead to positive effect on pain reduction $[9,10]$.

Stimulation of $\mathrm{C}$ fibers and A-delta fibers by electrical stimulation in favor of pain reduction requires high frequency and amplitude. The patients cannot tolerate this current or at most bear it for maximum 1 to 2 minutes. By simulating the action potential in these nerves, APS current can reduce the pain for long period of time without unpleasant or severe stimulation [10]. Electrical currents higher than the sensory threshold amplitude can be applied to motor nerves and muscle stimulation could reduce spasm and pain, enhance blood circulation, and eliminate edema. To achieve these goals, the muscle should be contracted but the electrical muscle contraction is not satisfying for patients. APS can increase endogenous narcotics and release adenosine and endor- phin through neuromatrix stimulation without muscle contraction, which leads to therapeutic effects and pain suppression [10]. Because of local stimulation quality, one side ions transportation, and bioelectrical features, APS is known as the most suitable current in traditional electrical science [10]. Treatment duration depends on type of electrical current and therapeutic aims. APS current can be applied in 3 time durations; depending on the treatment condition it can set as 4,8 , or 16 minutes, which is a short and proper time for therapeutic centers. APS therapy has no specific side effect and can be beneficial for several clinical goals such as massage, pain relief, and inflammation [10].

Because of the novelty of this therapeutic method, limited study has been conducted in this area. Eftekharsadat et al. reported that there was no notable difference between APS and interferential therapy in pain reduction of knee osteoarthritis patients [11]. Sepehri and Akbari reported the same results; no difference between APS and TENS in improvement of knee function and pain suppression [12].

The present study aimed to study and compare the effectiveness of 2 therapeutic methods, TENS and APS, in treatment of shoulder joint impairments. The results of this study can reveal the positive new therapeutic effects of APS such as no side effects, short time duration, faster results, and more pleasant sense rather than common treatments.

\section{Materials and Methods}

In this quasi-experimental study, 32 patients (women and men) with the range age of 18-45 were participated. The patients were referred from an orthopedic specialist with diagnosis of Chronic Mechanical Shoulder Pain (CMSP). The patients had no history of upper limb radicular pain, paralysis, trauma, surgery, and neuromuscular or cardiovascular disorders. The study protocol was approved by the Ethics Committee of Zahedan University of Medical Sciences.

The patients were classified in 4 groups; hyperactive with severe pain, hypoactive with severe pain, hyperactive with low pain, and hypoactive with low pain. The investigated outcomes were patients' activity, pain, shoulder joint mobility, function, instability, performance, and scapula position by America Surgeons Standardize Shoulder Assessment Form, Western Ontario Rotator Cuff Index, and Shoulder Dyskinesia [13-18].

For pain assessment, 3 pain states were evaluated; resting pain (0-6 score), night pain (0-6 score), and working 
pain (0-6 score). Shoulder mobility rate was measured in 3 movements of abduction in scapula (0-5), external rotation (0-2), and internal rotation (0-10). For shoulder girdle stability, the space between scapula and spinal cord was measured in two position; hands on the pelvic with the thumbs in backward position (0-2 score), and in $90^{\circ}$ abduction with shoulder in full internal rotation ( $0-2$ score).

Performing each task was asked in questions such as reaching the hands above the head, opposite axillary cavity, etc. Summation of all the points gives a number between 0 and 15 which is the shoulder function score. Scapula position was evaluated as the hands were placed alongside the body and score of 0-1 was given based on symmetry or asymmetry of scapula. Sum of all scores were considered as the shoulder joint performance rate [13-18].

Patients were randomly allocated into two group; APS or TENS. In the first group, monophasic APS current (Model APS-MK1 With frequency $150 \mathrm{~Hz}$, voltage 46 $\mathrm{V}$ and amplitude 0-24 mA) was used to reduce pain and for electrodes placement, negative (active) electrode was placed on the shoulder and the positive

(reference) electrode on the terminal part of pain region. Two electrodes distance was at least $25 \mathrm{~cm}$ and time duration was set for $16 \mathrm{~min}$ with dosage $1.2 \mathrm{~mA}$. Second group benefited from TENS current in burst mood, frequency of $50-100 \mathrm{~Hz}$ and pulse duration of 70 ms. Electrodes were placed on two sides of pain region and time duration of 20 minutes was selected. Patients in both group completed 6 therapeutic sessions [12] every day and reassessment was done at the end of the course.

The normality assumption of the data were checked by Shapiro-Wilk test. Also paired (within group) and Independent (between group) t tests were used. All statistical analyses were done by SPSS version 19. The significance level of all variables was considered at 0.05 .

\section{Results}

In Table 1, the general characteristics of subjects such as height, weight, and body mass index in the studied groups are presented. Regarding these characteristics, two study groups did not show significant differences $(\mathrm{P}>0.05)$. According to Table $2, \mathrm{t}$ test results showed average differences in study outcomes in day 1 and 6 and also the difference of day 1 and 6 in both group.

The Student t test analysis showed that the score of shoulder pain and movement is significant in the sixth day $(\mathrm{P}<0.05)$. The mean of APS group is higher than
TENS group, which means that APS was more effective in pain reduction and improvement of shoulder movement. The paired t test showed that difference of the first and sixth days was significant indicating the efficiency of both modalities in pain reduction and improvement of shoulder movement but the score rise in APS group was more prominent.

The paired $t$ test analysis showed that shoulder function score was not significant in the sixth day $(\mathrm{P}>0.05)$ and both modalities had the same role in shoulder function enhancement. The difference of first and sixth days was not significant $(\mathrm{P}>0.05)$. The Student $t$ test analysis showed that average score of APS group was higher than TENS group indicating the efficacy of APS.

Scores of scapula position was significant in the first day $(\mathrm{P}<0.05)$ indicating that the APS score is lower than TENS at the beginning of the study and the patients in TENS group entered the study with better function. On the sixth day, the difference was not significant $(\mathrm{P}>0.05)$, but the comparison between the first and sixth day was significant $(\mathrm{P}<0.05)$ indicating that APS was more effective than TENS with regard to the improvement of scapular position.

Shoulder performance score was not significant in the sixth day $(\mathrm{P}>0.05)$ but the APS score is more than TENS indicating the efficiency of APS. Comparison between the first and sixth days was significant $(\mathrm{P}<0.05)$ indicating the effectiveness of APS over TENS in improving the performance.

According to paired $\mathrm{t}$ test results, the average difference of pain, movement, stability, function, and performance of shoulder were significant in the first and sixth days in both groups $(\mathrm{P}<0.05)$, however, APS group showed major increases. Also mean difference of scapular position in the first and sixth days was not significant in TENS group ( $\mathrm{P}>0.05)$, but not in APS group $(\mathrm{P}<0.05)$ which shows higher effectiveness of APS in scapular position improvement.

\section{Discussion}

The results of the present study supports the effectiveness of APS in pain reduction and improvement of shoulder joint performance in patients with CMSP, however, there was no significant difference between groups in particular variables.

Different methods are used in physiotherapy program of patients with CMSP. In recent years, APS has been increasingly used as a useful method. Since APS does 
Table 1. General characteristics of the study subjects and descriptive information of the research variables.

\begin{tabular}{rccc}
\hline Variable & APS Group Mean \pm SD & TENS Group Mean \pm SD & P-Value \\
\hline Age $(\mathrm{y})$ & $33.1 \pm 9.28$ & $35.24 \pm 10.1$ & 0.528 \\
\hline Weight $(\mathrm{kg})$ & $63.92 \pm 8.13$ & $61.65 \pm 7.21$ & 0.479 \\
\hline Height $(\mathrm{cm})$ & $170.2 \pm 8.4$ & $168.3 \pm 7.25$ & 0.294 \\
\hline Body mass index $\left(\mathrm{kg} / \mathrm{m}^{2}\right)$ & $23.16 \pm 4.14$ & $23.78 \pm 3.25$ & 0.362 \\
\hline
\end{tabular}

PHYSICAL TREA $\pitchfork$ MENTS

Table 2. T-test results for average outcome differences on days 1 and 6 and the differences of days 1 and 6 results in both group.

\begin{tabular}{|c|c|c|c|c|c|c|c|c|c|}
\hline & \multicolumn{4}{|c|}{ TENS Group } & \multicolumn{4}{|c|}{ APS Group } & \multirow{2}{*}{$\begin{array}{c}\begin{array}{c}\text { Between } \\
\text { Group }\end{array} \\
\text { P-Value }\end{array}$} \\
\hline & Day 1 & Day 6 & $\begin{array}{l}\text { Difference of } \\
\text { the First and } \\
\text { Sixth Day }\end{array}$ & P-Value & $\begin{array}{l}\text { The First } \\
\text { Day }\end{array}$ & $\begin{array}{c}\text { The Sixth } \\
\text { Day }\end{array}$ & $\begin{array}{l}\text { Difference of } \\
\text { the First and } \\
\text { Sixth Day }\end{array}$ & P-Value & \\
\hline Pain & $11.5 \pm 2.7$ & $8.4 \pm 2.4$ & $3.1 \pm 0.3$ & 0.002 & $15.3 \pm 4.5$ & $8.8 \pm 3.0$ & $7.5 \pm 1.5$ & 0.003 & 0.000 \\
\hline Movement & $16.1 \pm 4.9$ & $18.2 \pm 5.4$ & $2.1 \pm 0.5$ & 0.001 & $11.9 \pm 3.9$ & $19.8 \pm 4.5$ & $7.9 \pm 0.6$ & 0.001 & 0.000 \\
\hline Stability & $1.8 \pm 1.1$ & $2.6 \pm 1.4$ & $0.8 \pm 0.3$ & 0.2 & $1.3 \pm 0.8$ & $3.2 \pm 1.1$ & $1.9 \pm 0.3$ & 0.4 & 0.000 \\
\hline Function & $9.5 \pm 2.1$ & $12 \pm 2.8$ & $2.5 \pm 0.7$ & 0.07 & $8.1 \pm 1.1$ & $11.7 \pm 1.9$ & $3.6 \pm 0.8$ & 0.06 & 0.000 \\
\hline Scapula position & $0.73 \pm 0.4$ & $0.80 \pm .0 .43$ & $0.07 \pm 0.03$ & 0.09 & $0.56 \pm 0.3$ & $0.9 \pm 042$ & $0.34 \pm 0.12$ & 0.06 & 0.000 \\
\hline Performance & $48.4 \pm 7.9$ & $53.9 \pm 9.0$ & $5.5 \pm 1.1$ & 0.08 & $34.7 \pm 7.9$ & $54.2 \pm 8.6$ & $0.7 \pm 19.5$ & 0.04 & 0.000 \\
\hline
\end{tabular}

PHYSICAL TREA \MENTS

not stimulate skin and senses, it is offered as a good treatment for pain relief. This modality brings about its useful effects through improving blood circulation and extracting the painful metabolites from pain locus [19]. Devices, which are capable of producing APS waves can generate various and specific low-frequency currents. Such currents are different in terms of period and intensity. Generally, three important types of APS waves have been introduced for medical applications. In one type, the painful locus is stimulated with a current equal to sensation threshold for 16 minutes, in another type the placebo effect of electrical stimulation without any current is applied, and in the third type, a current set to the highest bearable intensity is applied to the pain locus [23].

Shariatpanahi et al. study revealed that APS therapy can reduce pain in patients suffering from musculoskeletal pain [20]. In Toopchizadeh et al. research, there was no significant difference between TENS and APS in knee osteoarthritis treatment [8]. Rahimi et al. arranged a study, which showed no specific difference between APS and routine physical therapy in patients with knee osteoarthritis. According to these findings, APS is rec- ommended for reduction of knee pain and swelling that could be beneficial for knee osteoarthritis [21].

The presented study supports the effectiveness of APS in pain suppression and shoulder joint function improvement. There are few studies about mechanical shoulder pain which makes it difficult to compare the presented study with previous studies. Zizic et al. investigated a comprehensive study in multiple centers on 78 patients with knee osteoarthritis, which was a double blind randomized control trial. They evaluated 3 primary outcomes; pain, function, and general assessment as well as 6 secondary outcomes; joint range of motion, morning stiffness, tenderness, swelling, knee periphery, and walking duration in 4 weeks by a physician. Patients were treated by pulse electrical stimulation and placebo, which leads to significant improvement of pain and function but no specific difference was seen in tenderness, swelling, and walking duration [22].

Berger utilized 3 method of TENS, APS, and placebo on pain and swelling of patients with knee osteoarthritis. The results showed the efficacy of 8 minutes APS 
administration on knee pain and swelling reduction [23]. Papendorp studied the effects of APS therapy on 285 patients with chronic pain, which proved significant subjective and objective improvement of patients' movement and pain [6].

There are some possible mechanisms for medical benefits of APS and TENS techniques. It has been clinically shown that there are neurohormonal changes following APS therapy, which have a therapeutic effect on the biochemical balance in the treated area. Thus, when the physiological functions are impaired due to a breakdown in biochemistry and nerve function, it is feasible to attempt to reactivate those physiological functions electronically by using APS therapy. APS leads to an increase in specific neurohormones such as melatonin and leu-enkephalin, which reduces anxiety, pain, and sleep disorders. This achievement is gained without creating any clinically relevant changes in serotonin or cortisol concentrations, which are important hormones to maintain other important neural functions. It also creates an increase of oxygen around the positive electrode by breaking down electrolytes $[6,23]$.

Akbari, Forough, and Berger et al. reported sustainable signs of improvement one month after termination of therapy [19, 23]. Akbari and Forough reported positive effect of action potential stimulation on knee pain [23]. Pyszora et al. reported the positive effect of APS on chronic pain [9]. Johnson et al. introduced the electrical stimulation as a proper treatment to cure musculoskeletal pains [24]. Similarly, the our study results are in line with the findings of Zizic et al. and van Papendrop et al studies. The mentioned studies have attributed the analgesic effects of APS to higher secretion of beta-endorphins and leu-enkephalin and improved ATP release $[6,22]$.

With regard to the study results, various types of APS play similar roles in alleviating symptoms and improving function of patients with mild to moderate chronic mechanical shoulder pain, thus, applying any type of such treatments along with other non-drug therapies can be effective in improving the problems of such patients.

APS is a useful current for pain reduction and improvement of shoulder complex function at a short duration. Our results showed that APS current is useful for mechanical injuries, including shoulder pain.

\section{Acknowledgements}

The authors of this study would like to express their sincere thanks to colleagues in Physical Therapy Depart- ment of Khatam Hospital and Razmju Moghadam Clinic and appreciate all the students who participated in this project. The current research hasn't received any financial support.

\section{Conflict of Interest}

The author declared no conflict of interests.

\section{References}

[1] Levagie PK, Norkin CC. Joint structure and function. $3^{\text {rd }}$ ed. London: Jaypee Brothers; 2002..

[2] Kessler RA. Management of common musculoskeletal disorders. Physical therapy principles and methods. $3^{\text {rd }}$ ed. Philadelphia: Lippincott. 1996.

[3] Rene C. Shoulder pain syndrome. $1^{\text {st }}$ ed. Balitmore: Philadelphia; 1999.

[4] Maitland G. Practical orthopedic medicine. $1^{\text {st }}$ ed. London: Buller Words; 2003

[5] Carr AJ. Beyond disability: Measuring the social and personal consequences of osteoarthritis. Osteoarthritis and Cartilage. 1999; 7(2):230-8. doi: 10.1053/joca.1998.0154

[6] Papendrop DH, Kruger MC, Maritz C, Dippenaar NG. Action potential simulation therapy: Self assessment by 285 patients with chronic pain. Geneeskunde. 2000; 4(2):18-24

[7] Fengler RKB, Jacobs JWG, Bac M, van Wijck AJM, van Meeteren NLU. Action Potential Simulation (APS) in patients with Fibromyalgia Syndrome (FMS): A controlled single subject experimental design. Clinical Rheumatology. 2006; 26(3):322-9. doi: 10.1007/s10067-006-0297-z

[8] Toopchizadeh V, Babaei-Ghazani A, Eftekhar Sadat B. [Comparison of therapeutic effects of Action Potential Simulation (APS) and Transcutaneous Tlectrical Nerve Stimulation (TENS) in knee osteoarthritis (Persian)]. Quarterly Medical Journal of Tabriz University of Medical Sciences. 2013; 35(3):32-39.

[9] Pyszora A, Krajnik M, Adamczyk A, Graczyk M, Budzynski J, Zylicz Z, et al. Analgesic efficacy of APS (Action Potential Simulation); Pilot study of the patients with chronic pain due to musculoskeletal disorders. Advances in Palliative Medicine. 2007; 6(1):13-16.

[10] Osthuizen J, MCH, Wet E. Neurohormonal consequences of APS therapy. British Journal of Physiotherapy. 2011; 155:1013-1020.

[11] Eftekharsadat B, Babaei-Ghazani A, Habibzadeh A, Kolahi B. Efficacy of action potential simulation and interferential therapy in the rehabilitation of patients with knee osteoarthritis. Therapeutic Advances in Musculoskeletal Disease. 2015; 7(3):67-75. doi: 10.1177/1759720x15575724

[12] Sepehri R, Akbari M. [The effects of action potential stimulation on pain, swelling and function of patients with knee 
osteoarthritis (Persian)]. Zahedan Journal of Research in Medical Sciences. 2012; 14(6):12-16.

[13] Kibler WB. The role of the scapula in athletic shoulder function. American Journal of Sports Medicine. 1998; 26(2):32537. doi: $10.1177 / 03635465980260022801$

[14] Davies GY, Dickoff-Hoffman S. Neuromuscular testing and rehabilitation of the shoulder complex. Journal of Orthopaedic \& Sports Physical Therapy. 1993; 18(2):449-58. doi: 10.2519/jospt.1993.18.2.449

[15] Barrett WP, Franklin JL, Jackins SE, Wyss CR, Matsen FA. Total shoulder arthroplasty. Journal of Bone \& Joint Surgery. 1987; 69(6):865-72. doi: 10.2106/00004623-198769060-00011

[16] Franklin JL, Barrett WP, Jackins SE, Matsen FA. Glenoid loosening in total shoulder arthroplasty. Journal of Arthroplasty. 1988; 3(1):39-46. doi: 10.1016/s0883-5403(88)80051-2

[17] Kirkley A, Alvarez C, Griffin S. The development and evaluation of a disease-specific quality-of-life questionnaire for disorders of the rotator cuff: The western Ontario rotator cuff index. Clinical Journal of Sport Medicine. 2003; 13(2):8492. doi: 10.1097/00042752-200303000-00004

[18] Rubin B. Core based functional rehabilitation of the shoulder. Paper presented at: The $17^{\text {th }}$ Annual San Diego Shoulder Meeting; 2000 June 14-17; La Jolla, California.

[19] Akbari M, Forough B . [Comparison of the effect of APS and TENS in reduction of pain and function improvement of patients with mild to moderate ostheoarthritis of the knee (Persian)]. Annals of Military and Health Sciences Research. 2005; 3(4):659-663

[20] Shariatpanahi Sh, Mehdi Barzi D. [Effect of action potential simulation therapy in reduction of musculoskeletal pain (Persian)]. Daneshvar Medicine. 2007; 77:23-26.

[21] Rahimi A, Mohammadhossein F, Delnavaz M. [A comparative study on the Action Potential Simulation (APS) therapy and the routine physiotherapy protocol on pain and swelling reduction knee osteoarthritisin erderly people (Persian)] The Scientific Journal of Rehabilitation Medicine. 2012; 1(1):16-25.

[22] Zizic TM, Hoffman KC, Holt PA, Hungerford DS, O'Dell JR, Jacobs MA, et al. The treatment of osteoarthritis of the knee with pulsed electrical stimulation. Journal of rheumatology. 1995; 22(9):1757-61. PMID: 8523357

[23] Berger L. Introducing action potential currents. South Philadelphia: Sage; 2002.

[24] Johnson M, Martinson M. Efficacy of electrical nerve stimulation for chronic musculoskeletal pain: A meta-analysis of randomized controlled trials. Pain. 2007; 130(1):157-65. doi: 10.1016/j.pain.2007.02.007 